\title{
Spatially resolving edge states of chiral graphene nanoribbons
}

\author{
Chenggang Tao ${ }^{1,2} \uparrow$, Liying Jiao ${ }^{3 \dagger}$, Oleg V. Yazyev ${ }^{1,2 \dagger}$, Yen-Chia Chen ${ }^{1,2}$, Juanjuan Feng ${ }^{1,4}$, \\ Xiaowei Zhang ${ }^{1,2}$, Rodrigo B. Capaz ${ }^{1,5}$, James M. Tour ${ }^{6}$, Alex Zett $\left.\right|^{1,2}$, Steven G. Louie ${ }^{1,2}$, Hongjie Dai ${ }^{3}$ \\ and Michael F. Crommie ${ }^{1,2 \star}$
}

A central question in the field of graphene-related research is how graphene behaves when it is patterned at the nanometre scale with different edge geometries. A fundamental shape relevant to this question is the graphene nanoribbon (GNR), a narrow strip of graphene that can have different chirality depending on the angle at which it is cut. Such GNRs have been predicted to exhibit a wide range of behaviour, including tunable energy gaps ${ }^{1,2}$ and the presence of one-dimensional (1D) edge states ${ }^{3-5}$ with unusual magnetic structure ${ }^{6,7}$. Most GNRs measured up to now have been characterized by means of their electrical conductivity, leaving the relationship between electronic structure and local atomic geometry unclear ${ }^{8-10}$. Here we present a sub-nanometre-resolved scanning tunnelling microscopy (STM) and spectroscopy (STS) study of GNRs that allows us to examine how GNR electronic structure depends on the chirality of atomically well-defined GNR edges. The GNRs used here were chemically synthesized using carbon nanotube (CNT) unzipping methods that allow flexible variation of GNR width, length, chirality, and substrate ${ }^{11,12}$. Our STS measurements reveal the presence of 1D GNR edge states, the behaviour of which matches theoretical expectations for GNRs of similar width and chirality, including width-dependent energy splitting of the GNR edge state.

The chirality of a GNR is characterized by a chiral vector $(n, m)$ or, equivalently, by chiral angle $\theta$, as shown in Fig. la. GNRs having different widths and chiralities were deposited on a clean $\mathrm{Au}(111)$ surface and measured using STM. Figure $1 \mathrm{~b}$ shows a room temperature image of a single monolayer GNR (GNR height is determined from linescans, such as that shown in Fig. $1 \mathrm{~b}$ inset; some multilayer GNRs were observed, but we focus here on monolayer GNRs). The GNR of Fig. 1b has a width of $23.1 \mathrm{~nm}$, a length greater than $600 \mathrm{~nm}$, and exhibits straight, atomically smooth edges (the highest quality GNR edges, such as those shown in Figs 1 and 2, were observed in GNRs synthesized as in ref. 11). Such GNRs are seen to have a 'bright stripe' running along each edge.

This stripe marks a region of curvature near the terminal edge of the GNR that has a maximum extension of $\sim 3 \AA$ above the mid-plane terrace of the GNR and a width of $\sim 30 \AA$ (see line scan in Fig. 1b inset). Such edge-curvature was observed for all high-quality GNRs examined in this study (more than 150, including GNRs deposited onto a $\mathrm{Ru}(0001)$ surface). This
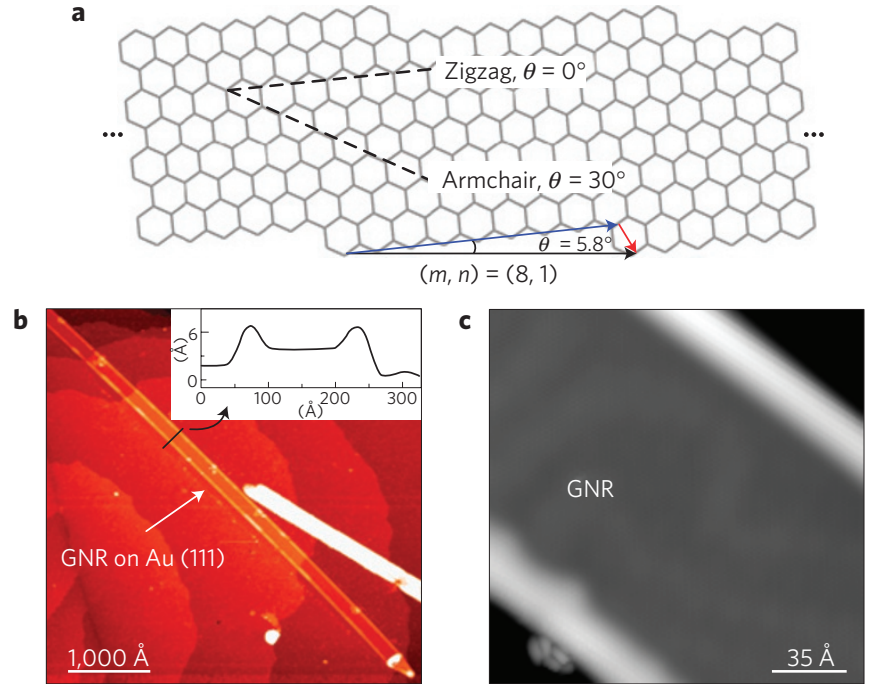

Figure 1 | Topography of graphene nanoribbons (GNRs) on Au(111). a, A schematic drawing of an $(8,1)$ GNR. The chiral vector $(n, m)$ connecting crystallographically equivalent sites along the edge defines the edge orientation of the GNR (black arrow). The blue and red arrows are the projections of the $(8,1)$ vector onto the basis vectors of the graphene lattice. Zigzag and armchair edges have corresponding chiral angles of $\theta=0^{\circ}$ and $\theta=30^{\circ}$, respectively, whereas the $(8,1)$ edge has an chiral angle of $\theta=5.8^{\circ} \cdot \mathbf{b}$, Constant-current STM image of a monolayer GNR on $\mathrm{Au}(111)$ at room temperature $\left(V_{\mathrm{s}}=1.5 \mathrm{~V}, I=100 \mathrm{pA}\right)$. Inset shows the indicated line profile. $\mathbf{c}$, Higher resolution STM image of a GNR at $T=7 \mathrm{~K}$ $\left(V_{\mathrm{s}}=0.2 \mathrm{~V}, I=30 \mathrm{pA}\right.$, greyscale height map).

is reminiscent of curved edge structures observed previously near graphite step-edges ${ }^{13}$. We rule out that these GNRs are collapsed nanotubes by virtue of the measured ratio (observed to be $\pi$ ) of GNR width to nanotube height for partially unzipped CNTs. We further rule out that the curved GNR edges observed here are folded graphene boundaries by means of a detailed comparison of terminal curved edges and actual folded edges (see Supplementary Information). Low-temperature STM images (Figs 1c and 2a) show finer structure in both the interior GNR terrace and the edge region. Figure 2a, for example, shows the atomically-resolved edge

\footnotetext{
'Department of Physics, University of California at Berkeley, Berkeley, California 94720, USA, ${ }^{2}$ Materials Science Division and Chemical Science Division, Lawrence Berkeley National Laboratory, Berkeley, California 94720, USA, ${ }^{3}$ Department of Chemistry and Laboratory for Advanced Materials, Stanford University, Stanford, California 94305, USA, ${ }^{4}$ School of Physical Science and Technology, Lanzhou University, Lanzhou, Gansu 730000, China, ${ }^{5}$ Instituto de Física, Universidade Federal do Rio de Janeiro, Caixa Postal 68528, Rio de Janeiro, RJ 21941-972, Brazil, ${ }^{6}$ Department of Chemistry, and Smalley Institute for Nanoscale Science and Technology, Rice University, Texas 77005, USA. †These authors contributed equally to this work. *e-mail: crommie@berkeley.edu.
} 


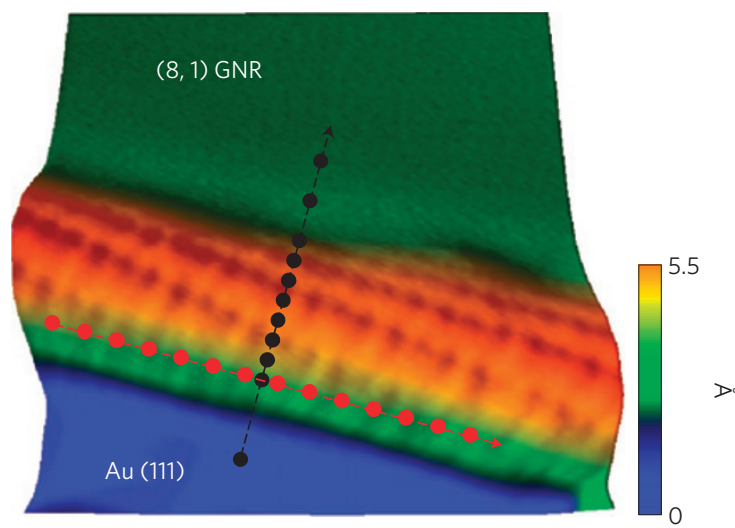

$48 \AA \times 48 \AA$

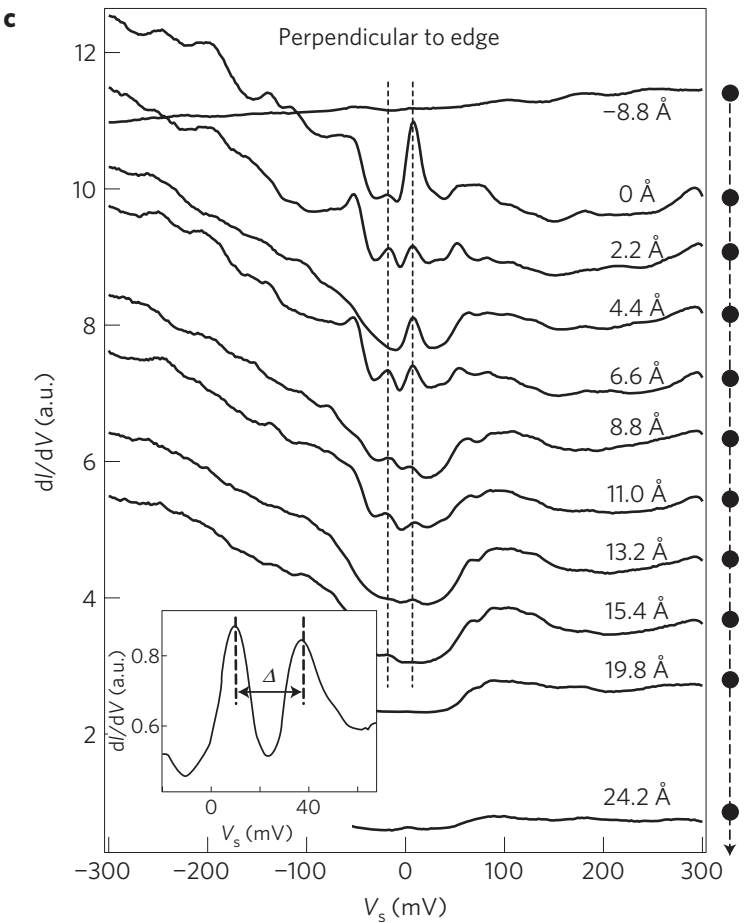

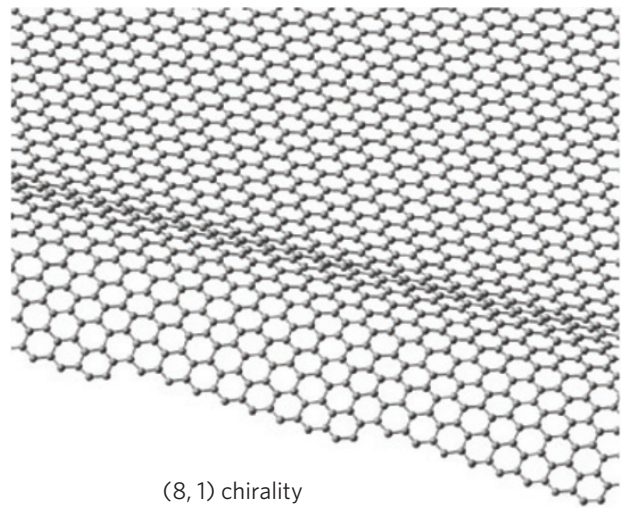

d

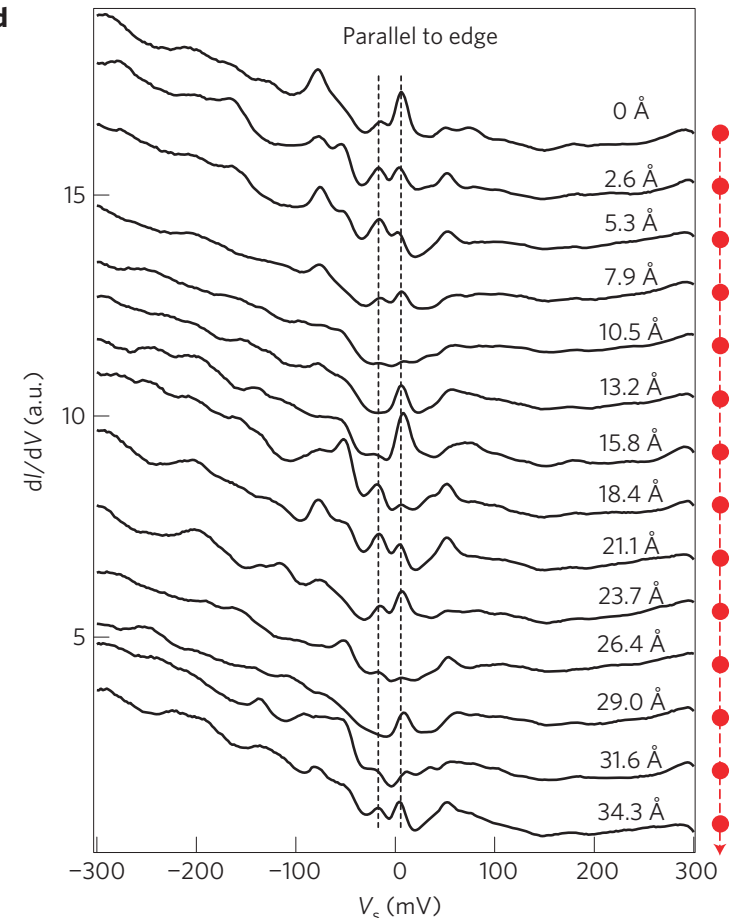

Figure 2 | Edge states of GNRs. a, Atomically-resolved topography of the terminal edge of an $(8,1)$ GNR with measured width of $19.5 \pm 0.4$ nm

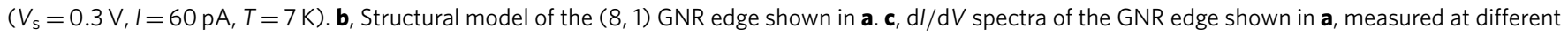

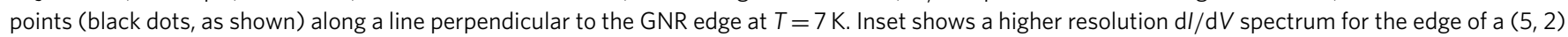
GNR with width of $15.6 \pm 0.1 \mathrm{~nm}$ (initial tunnelling parameters $V_{\mathrm{s}}=0.15 \mathrm{~V}, I=50 \mathrm{pA}$; wiggle voltage $V_{\text {r.m.s. }}=2 \mathrm{mV}$ ). The dashed lines are guides to the eye. $\mathbf{d}, \mathrm{d} / \mathrm{d} V$ spectra measured at different points (red dots, as shown) along a line parallel to the GNR edge shown in a at $T=7 \mathrm{~K}$ (initial tunnelling parameters for $\mathbf{c}$ and $\mathbf{d}$ are $V_{\mathrm{s}}=0.3 \mathrm{~V}, I=50 \mathrm{pA}$; wiggle voltage $V_{\text {r.m.s. }}=5 \mathrm{mV}$ ).

region of a monolayer GNR and clearly exhibits how the periodic graphene sheet of the GNR terminates cleanly and with atomic order at the gold surface.

Such high-resolution images allow us to experimentally determine the chirality of GNRs, and to create structural models of observed edge regions. In Fig. 2a, for example, we see rows of protrusions (with a spacing, $\sim 2.5 \AA$, equivalent to the graphene lattice) near the edge of a GNR having a width of $19.5 \pm 0.4 \mathrm{~nm}$. By comparing this row orientation with the orientation of the GNR terminal edge we are able to extract the GNR chirality (details in Supplementary Information). The GNR shown in Fig. 2a has an $(8,1)$ chirality (equivalent to $\theta=5.8^{\circ}$ ), and the resulting structural model for this GNR is shown in Fig. 2b. We find the distribution of GNR chiralities to be essentially random. This is consistent with our structural data, which indicates that the CNT unzipping direction is very close to the axial direction of the precursor CNTs (see
Supplementary Information), as well as the fact that the precursor CNTs have a broad chirality distribution ${ }^{14}$.

We explored the local electronic structure of GNR edges using STS, in which $\mathrm{d} I / \mathrm{d} V$ measurement reflects the energy-resolved local density of states (LDOS) of a GNR. Figure $2 \mathrm{c}, \mathrm{d}$ shows $\mathrm{d} I / \mathrm{d} V$ spectra obtained at different positions (as marked) near the edge of the $(8,1)$ GNR pictured in Fig. $2 \mathrm{a}$. $\mathrm{d} I / \mathrm{d} V$ spectra measured within $24 \AA$ of the GNR edge typically show a broad gap-like feature having an energy width of $\sim 130 \mathrm{meV}$. This is very similar to the behaviour observed in the middle of large-scale graphene sheets, and is attributed to the onset of phonon-assisted inelastic electron tunnelling ${ }^{15}$ for $|E| \geq 65 \mathrm{meV}$. This feature disappears further into the interior of the GNR, as expected, because of increased tunnelling to the Au substrate ${ }^{16}$. Very close to the GNR edge, however, we observe further features in the spectra. The most dominant of these features are two peaks that rise up within the elastic tunnelling 
region (that is at energies below the phonon-assisted inelastic onset) and which straddle zero bias. For the GNR shown in Fig. 2a (which has a width of $19.5 \pm 0.4 \mathrm{~nm}$ ) the two peaks are separated in energy by a splitting of $\Delta=23.8 \pm 3.2 \mathrm{meV}$. Similar energy-split edge-state peaks have been observed in all the clean chiral GNRs that we investigated spectroscopically at low temperature. For example, the inset to Fig. 2c shows a higher resolution spectrum exhibiting energy-split edge-state peaks for a $(5,2)$ GNR having a width of $15.6 \mathrm{~nm}$ and an energy splitting of $\Delta=27.6 \pm 1.0 \mathrm{meV}$. The two edge-state peaks are often asymmetric in intensity (depending on specific location in the GNR edge region), and their mid-point is often slightly offset from $V_{s}=0$ (within a range of $\pm 20 \mathrm{meV}$ ). As seen in the spectra of Fig. $2 c$, the amplitude of the peaks grows as one moves closer to the terminal edge of the GNR, before falling abruptly to zero as the carbon/gold terminus is crossed. The spatial dependence of the edge-state peak amplitude as one moves perpendicular from the GNR edge is plotted in Fig. 3a and shows exponential behaviour. The edge-state spectra also vary as one moves parallel to the GNR edge, as shown in Fig. $2 \mathrm{~d}$. The parallel dependence of the edge-state peak amplitude is plotted in Fig. 3b, and oscillates with an approximate $20 \AA$ period, corresponding closely to the $21 \AA$ periodicity of an $(8,1)$ edge.

We have also characterized monolayer GNRs having different chiralities and widths (the lengths of the GNRs used in these measurements are greater than $500 \mathrm{~nm}$ ). In Fig. $3 \mathrm{c}$, we plot the width dependence of the measured energy gap of GNR edge states for a broad range of chirality $\left(3.7^{\circ}<\theta<16.1^{\circ}\right)$. The measured edge-state energy splitting shows a clear inverse correlation with GNR width. Our gap values tend to be smaller than those observed previously for lithographically patterned GNRs (probably because of uncertainty in the edge structure of lithographically obtained GNRs; ref. 9).

The high quality of the atomically well-defined edge structures observed here allows us to quantitatively compare our experimental data to theoretical calculations of the electronic structure of chiral GNRs. We find that the spectroscopic features we observe correspond closely to the spatial- and energy-dependence predicted for 1D spin-polarized edge states coupled across the width of a chiral GNR. This behaviour is quite different from the properties observed previously for graphite step edges, armchair nanoribbons, and comparatively less ordered graphene platelet edges, where no magnetism-induced energy splitting has been seen ${ }^{17-20}$.

To compare our experimental data with theoretical predictions for GNRs, we used a Hubbard model Hamiltonian, solved selfconsistently in the mean-field approximation ${ }^{6}$, for an $(8,1)$ GNR having the same width as the actual $(8,1)$ GNR shown in Fig. 2a. The Hamiltonian:

$$
H=-t \sum_{\langle i j\rangle, \sigma}\left[c_{i \sigma}^{\dagger} c_{j \sigma}+\text { h.c. }\right]+U \sum_{i} n_{i \uparrow} n_{i \downarrow}
$$

consists of a one-orbital nearest-neighbour tight-binding Hamiltonian with an on-site Coulomb repulsion term. In this expression $c_{i \sigma}{ }^{\dagger}$ and $c_{j \sigma}$ are operators that create and annihilate an electron with spin $\sigma$ at the nearest-neighbour sites $i$ and $j$ respectively, $t=2.7 \mathrm{eV}$ is a hopping integral ${ }^{21}, n_{i \sigma}=c_{i \sigma}{ }^{\dagger} c_{i \sigma}$ is the spin-resolved electron density at site $i$, and $U$ is an on-site Coulomb repulsion. This GNR model is defined only by the $\pi$-bonding network. The terminal $\sigma$-bonds at the GNR edges are considered to be passivated and do not alter the $\pi$-system (this should, in general, correctly model a range of different possible edge-adsorbate bonding configurations ${ }^{7,22}$, including the likely oxygen-related functional group termination of our own GNRs; ref. 11). The out-of-plane curvature seen experimentally near GNR edges is not included in this model because the measured radii of curvature are sufficiently large $(>20 \AA)$ that they are not expected to significantly affect GNR electronic structure ${ }^{23}$ (we
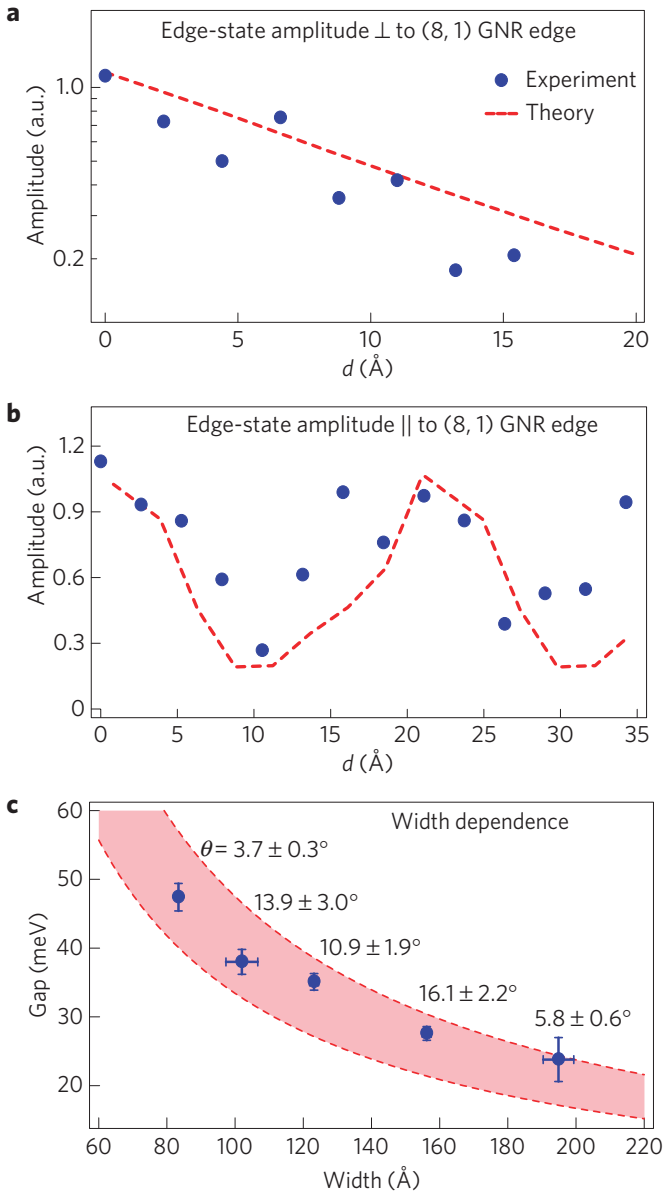

Figure 3 | Position- and width-dependent edge-state properties. a, Solid blue dots show experimental edge-state peak amplitude at points along a line perpendicular to the carbon/gold edge terminus (same positions as shown in Fig. 2c). Peak amplitude and energies were determined by fitting Lorentzian curves to the two peaks observed in the measured spectra at each location in Fig. $2 \mathrm{c}$ over the range $-30 \mathrm{mV}<V_{\mathrm{s}}<30 \mathrm{mV}$. The energy positions of these peaks were found to be $6.7 \pm 1.6 \mathrm{mV}$ and

$-17.2 \pm 2.2 \mathrm{mV}$. The positional dependence of the average peak amplitude of these two peaks is plotted. Error bars (shown when larger than plotted points) reflect the range of Lorentzian parameters that result in a good fit to the data. Dashed red line shows the calculated LDOS at locations spaced perpendicular to the edge terminus for an $(8,1)$ GNR (see text) at the energy of the DOS peak nearest the band-edge. Theoretical LDOS values include a single globally constant offset to model the added contribution from Au surface LDOS, and a single globally constant multiplicative factor to model the unknown total area of the STM tunnel junction. $\mathbf{b}$, Solid blue dots show experimental average edge-state peak amplitude (determined as in a) at locations spaced along a line parallel to the carbon/gold edge terminus (same positions as shown in Fig. $2 \mathrm{~d}$ ). Dashed red line shows the theoretical edge-state LDOS for an $(8,1)$ GNR at points parallel to the edge terminus (calculated as in $\mathbf{a}$ ). The edge-state LDOS amplitude oscillates parallel to the edge with a $21 \AA$ period. c, Width dependence of the edge-state energy gap of chiral GNRs. From left to right, the chiralities of experimentally measured GNRs are $(13,1),(3,1),(4,1),(5,2)$, and $(8,1)$ respectively, corresponding to a range of chiral angle $3.7^{\circ}<\theta<16.1^{\circ}$. Energy gaps determined by Lorentzian fits to $\mathrm{d} / \mathrm{d} V$ peaks (centre-to-centre width), error bars reflect standard deviation due to spatial variation in spectra. GNR width measured as distance between the GNR edge mid-heights on opposite sides, error bars reflect standard deviation due to spatial variation along GNR axis. The pink shaded area shows the predicted range of edge-state bandgaps as a function of width, evaluated for chiral angles in the range $0^{\circ}<\theta<15^{\circ}(U=0.5 t, t=2.7 \mathrm{eV}$; ref. 27). 
a

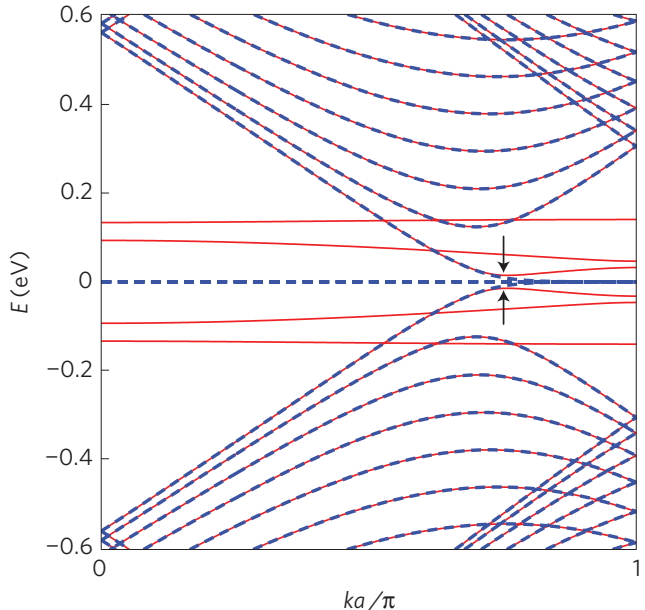

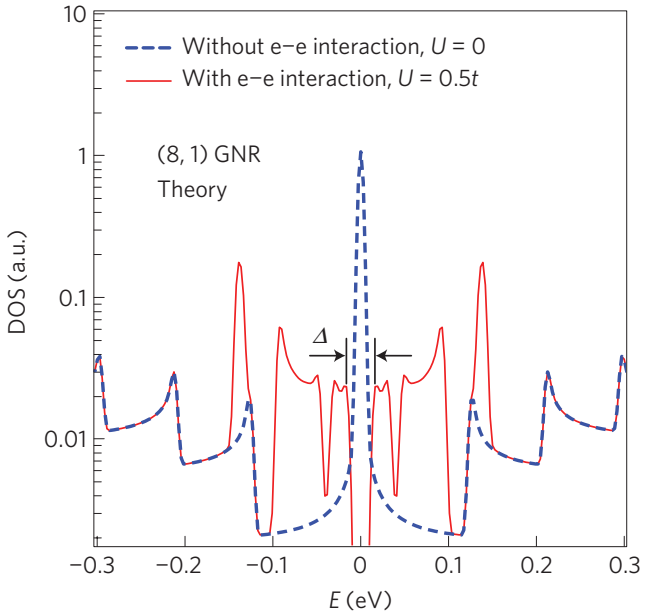

Figure 4 | Theoretical band structure and density of states (DOS) of a 20-nm-wide $(\mathbf{8}, \mathbf{1})$ GNR. a, Dashed blue line shows the calculated GNR electronic structure in the absence of electron-electron interactions $(U=0)$. Solid red line shows the calculated GNR electronic structure for $U=0.5 t(t=2.7 \mathrm{eV})$. Finite $U>0$ splits degenerate edge states at $E=0$ into spin-polarized bands, opening a bandgap (arrows). b, Dashed blue line shows the $(8,1)$ GNR DOS for the $U=0$ case in $\mathbf{a}$. The peak at $E=0$ is due to the degeneracy of edge states in the absence of electron-electron interactions. Solid red line shows the $(8,1)$ GNR DOS for $U=0.5 t$. The opening of the bandgap (arrows) reflects the predicted energy splitting due to the onset of magnetism in spin-polarized edge states for $U>0$, and compares favourably with the experimental data for the $(8,1)$ GNR of Fig. 2.

tested this conjecture by including the observed curvature in some calculations, and found that it has no significant effect-either from $\sigma-\pi$ coupling or from pseudofield effects-on the calculated GNR electronic structure). The effect of the gold substrate here is taken only as a charge reservoir that can slightly shift the location of $E_{\mathrm{F}}$ within the GNR band structure and reduce the magnitude of the effective $U$ parameter by means of electrostatic screening (the experimental charge-induced energy shifts seen here are within the range of charge-induced energy shifts observed previously for CNTs on Au; ref. 24).

We first calculated the GNR electronic structure for $U=0$, which effectively omits the electron-electron interactions responsible for the onset of magnetic correlations. This results in the theoretical band structure and density of states (DOS) shown in Fig. 4a, $\mathrm{b}$ (blue dashed lines). The finite width of the GNR leads to a family of subbands in the band structure, with no actual bandgap (Fig. 4a). A flat band at $E=0$ due to localized edge states leads to a strong van Hove singularity (that is, a peak) in the DOS at $E=0$ (Fig. 4 b). The DOS in this case does not resemble what is seen experimentally. We next calculated the $(8,1)$ GNR electronic structure for $U>0$. Here the electron-electron interactions lift the degeneracy of the edge states by causing ferromagnetic correlations to develop along the GNR edges and antiferromagnetic correlations to develop across the GNR. This leads to a spin-polarization of the edge states that splits the single low-energy peak seen in the $U=0$ DOS into a series of van Hove singularities, thus opening up a gap at $E=0$. Such behaviour is seen in the band structure and DOS of Fig. $4 \mathrm{a}, \mathrm{b}$ (solid red lines). We identify the lowest-energy pair of van Hove singularities with the pair of peaks observed experimentally near zero bias for GNR edges. We focus our experiment/theory comparison on the low-energy regime $(|E| \leq 65 \mathrm{meV})$ because higher energy experimental features are complicated by the onset of phonon-assisted inelastic tunnelling ${ }^{25}$ (the low-energy edge-state peaks, by contrast, do not have the characteristics of inelastic modes).

We find that our experimental spectroscopic edge-state data for the $(8,1)$ GNR is in agreement with model Hamiltonian calculations for $U=0.5 t$. The theoretical bandgap of $29 \mathrm{meV}$ is very close to the experimentally observed value of $23.8 \pm 3.2 \mathrm{meV}$ (the value of $U$ used here is lower than a value obtained previously from a first-principles calculation ${ }^{26}$, presumably because of screening from the gold substrate). Our experimentally observed energy-split spectroscopic peaks thus provide evidence for the formation of spin-polarized edge states in pristine GNRs (such splitting does not arise for the non-magnetic $U=0$ case described above). We are further able to compare the spatial dependence of the calculated edge states with the experimentally measured STS results. The dashed line in Fig. 3a shows the theoretical LDOS calculated at the energy of the low-energy edge-state peaks as one moves perpendicularly away from the GNR edge and into the $(8,1)$ GNR interior. The predicted exponential decay length of $\sim 12 \AA$ is in reasonable agreement with the experimental data. The variation seen in the calculated LDOS of the edge state in the direction parallel to the GNR edge also compares favourably with our experimental observations (Fig. 3b). The oscillation in edge-state amplitude is seen to arise from 'kinks' in the zigzag edge structure resulting from the chiral nature of the $(8,1)$ GNR edge (see the edge structure of Fig. 2b, each of the two dips in spectroscopic amplitude occurs at the location of a kink).

We are similarly able to compare the GNR width dependence of our experimentally measured edge-state gaps to theoretical calculations. As the measured GNRs having different widths also have different chiralities, we have calculated the theoretical edge-state gap versus width behaviour over the chirality range $0^{\circ}<\theta<15^{\circ}$ (it will be useful in the future to measure the local electronic properties of armchair GNRs $\left(\theta=30^{\circ}\right)$, which are predicted to have no edge states). The pink shaded region in Fig. $3 \mathrm{c}$ shows the results of our calculations, and compares favourably with our experimentally observed width-dependent edge-state gap. This provides strong evidence that the edge-state gap we observe experimentally is not a local effect, as might occur, say, in response to some unknown molecules bound to the GNR edge, but rather depends on the full GNR electronic structure, including interaction between the edges.

\section{Methods}

The GNRs shown in this study were produced by unzipping carbon nanotubes ${ }^{11}$ GNRs were deposited onto clean Au(111) surfaces using a spin-coating method. $\mathrm{Au}(111)$ substrates were first cleaned by sputtering and annealing in ultra-high vacuum (UHV) before spin-coating. The samples were then transferred into the UHV chamber of our STM system (base pressure $\sim 2.0 \times 10^{-10}$ torr). After heat treatment up to $500^{\circ} \mathrm{C}$ in UHV, the samples were directly transferred onto the STM stage in the same chamber for measurements. 
STM measurements were performed using a home-built STM held at low temperature $(T=7 \mathrm{~K})$ for maximum spatial and spectroscopic resolution. STM topography was obtained in constant-current mode using a PtIr tip, and $\mathrm{d} I / \mathrm{d} V$ spectra were measured using lock-in detection of the a.c. tunnelling current driven by a $451 \mathrm{~Hz}, 1-5 \mathrm{mV}$ (r.m.s.) signal added to the junction bias (the sample potential referenced to the tip) under open-loop conditions. We also performed large-scale topographic surveys of GNR samples before low-temperature measurement. This was done in an Omicron variable temperature STM in UHV at room temperature. The three large-scale topographic STM images shown (Fig. $1 \mathrm{~b}$ and Supplementary Figs S1a,b) were obtained in the Omicron STM at room temperature.

Received 4 January 2011; accepted 30 March 2011; published online 8 May 2011; corrected online 11 May 2011

\section{References}

1. Son, Y. W., Cohen, M. L. \& Louie, S. G. Energy gaps in graphene nanoribbons Phys. Rev. Lett. 97, 216803 (2006)

2. Ezawa, M. Peculiar width dependence of the electronic properties of carbon nanoribbons. Phys. Rev. B 73, 045432 (2006).

3. Nakada, K., Fujita, M., Dresselhaus, G. \& Dresselhaus, M. S. Edge state in graphene ribbons: Nanometer size effect and edge shape dependence. Phys. Rev. B 54, 17954-17961 (1996).

4. Akhmerov, A. R. \& Beenakker, C. W. J. Boundary conditions for Dirac fermions on a terminated honeycomb lattice. Phys. Rev. B 77, 085423 (2008).

5. Wimmer, M., Akhmerov, A. R. \& Guinea, F. Robustness of edge states in graphene quantum dots. Phys. Rev. B 82, 045409 (2010).

6. Fujita, M., Wakabayashi, K., Nakada, K. \& Kusakabe, K. Peculiar localized state at zigzag graphite edge. J. Phys. Soc. Jpn 65, 1920-1923 (1996).

7. Son, Y. W., Cohen, M. L. \& Louie, S. G. Half-metallic graphene nanoribbons. Nature 444, 347-349 (2006).

8. Chen, Z. H., Lin, Y. M., Rooks, M. J. \& Avouris, P. Graphene nano-ribbon electronics. Physica E 40, 228-232 (2007).

9. Han, M. Y., Ozyilmaz, B., Zhang, Y. B. \& Kim, P. Energy band-gap engineering of graphene nanoribbons. Phys. Rev. Lett. 98, 206805 (2007).

10. Li, X. L., Wang, X. R., Zhang, L., Lee, S. W. \& Dai, H. J. Chemically derived, ultrasmooth graphene nanoribbon semiconductors. Science 319, 1229-1232 (2008).

11. Jiao, L. Y., Wang, X. R., Diankov, G., Wang, H. L. \& Dai, H. J. Facile synthesis of high-quality graphene nanoribbons. Nature Nanotech. 5, 321-325 (2010).

12. Kosynkin, D. V. et al. Longitudinal unzipping of carbon nanotubes to form graphene nanoribbons. Nature 458, 872-875 (2009).

13. Atamny, F., Fassler, T. F., Baiker, A. \& Schlogl, R. On the imaging mechanism of monatomic steps in graphite. Appl. Phys. A 71, 441-447 (2000).

14. Bachilo, S. M. et al. Structure-assigned optical spectra of single-walled carbon nanotubes. Science 298, 2361-2366 (2002).

15. Zhang, Y. B. et al. Giant phonon-induced conductance in scanning tunnelling spectroscopy of gate-tunable graphene. Nature Phys. 4, 627-630 (2008).

16. Levy, N. et al. Strain-induced pseudo-magnetic fields greater than 300 Tesla in graphene nanobubbles. Science 329, 544-547 (2010).

17. Klusek, Z. et al. Observations of local electron states on the edges of the circular pits on hydrogen-etched graphite surface by scanning tunnelling spectroscopy. Appl. Surf. Sci. 161, 508-514 (2000).
18. Kobayashi, Y., Fukui, K., Enoki, T., Kusakabe, K. \& Kaburagi, Y. Observation of zigzag and armchair edges of graphite using scanning tunnelling microscopy and spectroscopy. Phys. Rev. B 71, 193406 (2005).

19. Ritter, K. A. \& Lyding, J. W. The influence of edge structure on the electronic properties of graphene quantum dots and nanoribbons. Nature Mater. 8, 235-242 (2009).

20. Cai, J. M. et al. Atomically precise bottom-up fabrication of graphene nanoribbons. Nature 466, 470-473 (2010)

21. Reich, S., Maultzsch, J., Thomsen, C. \& Ordejon, P. Tight-binding description of graphene. Phys. Rev. B 66, 035412 (2002).

22. Hod, O., Barone, V., Peralta, J. E. \& Scuseria, G. E. Enhanced half-metallicity in edge-oxidized zigzag graphene nanoribbons. Nano Lett. 7, 2295-2299 (2007).

23. Blase, X., Benedict, L. X., Shirley, E. L. \& Louie, S. G. Hybridization effects and metallicity in small radius carbon nanotubes. Phys. Rev. Lett. 72, 1878-1881 (1994).

24. Cui, X. D., Freitag, M., Martel, R., Brus, L. \& Avouris, P. Controlling energy-level alignments at carbon nanotube/Au contacts. Nano Lett. 3, 783-787 (2003)

25. Brar, V. W. et al. Observation of carrier-density-dependent many-body effects in graphene via tunneling spectroscopy. Phys. Rev. Lett. 104, 036805 (2010).

26. Yazyev, O. V. Magnetism in disordered graphene and irradiated graphite. Phys. Rev. Lett. 101, 037203 (2008).

27. Yazyev, O. V., Capaz, R. B. \& Louie, S. G. Theory of magnetic edge states in chiral graphene nanoribbons. Preprint at http://arxiv.org/abs/1102.4886 (2011).

\section{Acknowledgements}

Research supported by the Office of Naval Research Multidisciplinary University Research Initiative (MURI) award nos. N00014-09-1-1066 and N00014-09-1-1064 (GNR sample preparation and characterization, GNR modelling), by the Helios Solar Energy Research Center, which is supported by the Director, Office of Science, Office of Basic Energy Sciences of the US Department of Energy under contract no. DE-AC02-05CH11231 (STM instrumentation development and measurements), by the National Science Foundation award no. DMR-0705941 (software development to numerically simulate electron correlation effects), by Intel (development of GNR synthesis techniques), and by MARCO MSD (preliminary GNR screening through use of AFM and transport methods). R.B.C. acknowledges financial support from Brazilian agencies CNPq, CAPES, FAPERJ, and INCT.

\section{Author contributions}

C.T., L.J., Y-C.C., J.F., X.Z. and M.F.C. performed STM measurements and analysed STM data. L.J., C.T., Y-C.C., J.F., X.Z., J.M.T. and H.D. prepared samples. A.Z. assisted in structural analysis. O.V.Y., R.B.C. and S.G.L. carried out theoretical GNR simulations. All authors discussed the results and wrote the paper.

\section{Additional information}

The authors declare no competing financial interests. Supplementary information accompanies this paper on www.nature.com/naturephysics. Reprints and permissions information is available online at http://www.nature.com/reprints. Correspondence and requests for materials should be addressed to M.F.C. 